\title{
RESEARCH NOTES \\ RANDOM PROCESSES WITH CONVEX COORDINATES ON TRIANGULAR GRAPHS
}

\author{
J.N. BOYD \\ P.N. RAYCHOWDHURY \\ Department of Mathematical Sciences \\ Virginia Commonwealth University \\ Richmond Virginia 23284-2014 U.S.A.
}

(Received September 28, 1994)

\begin{abstract}
Probabilities for reaching specified destinations and expectation values for lengths for random walks on triangular arrays of points and edges are computed. Probabilities and expectation values are given as functions of the convex (barycentric) coordinates of the starting point.
\end{abstract}

KEYWORDS AND PHRASES. Random walk, expectation value, trap, convex (barycentric) coordinates.

1991 AMS SUBJECT CLASSIFICATION CODES. Primary 60J15, Secondary 60J05.0

\section{INTRODUCTION.}

Barycentric coordinates were introduced into mathematics by the geometer A.F. Möbius (1790-1868). Suppose that $\mathrm{V}_{1}, \mathrm{~V}_{2}, \mathrm{~V}_{3}, \ldots, \mathrm{V}_{n}, \mathrm{~V}_{n+1}$ are the linearly independent vertices of an $\mathrm{n}$ dimensional Euclidean simplex. The barycentric coordinates of point $P$ in the Euclidian space are given by the unique $(n+1)$-tuple of real numbers $\alpha_{i}$ such that $P=\sum_{i=1}^{n+1} \alpha_{i} V_{i}$ with $\sum_{i=1}^{n+1} \alpha_{i}=1$. [1]

The points of the closed simplex $S_{n}=\mathrm{V}_{1} \mathrm{~V}_{2} \mathrm{~V}_{3} \ldots \mathrm{V}_{n} \mathrm{~V}_{n+1}$ are those points for which all barycentric coordinates are nonnegative. The barycentric coordinates of vertex $\mathrm{V}_{\mathrm{s}}$ are given by $\alpha_{\mathbf{i}}=1, \alpha_{j}=0$ for $\mathrm{j} \neq \mathrm{i}$. In our paper, we are concerned only with points of the closed simplex, and we will refer to $\left(\alpha_{1}, \alpha_{2}, \alpha_{3}, \ldots, \alpha_{n}\right)$ as the convex coordinates of $P \epsilon S_{n}$ with respect to the vertices in the order $\mathrm{V}_{1}, \mathrm{~V}_{2}, \mathrm{~V}_{3}, \ldots, \mathrm{V}_{n}, \mathrm{~V}_{n+1}$. However, our primary concern will be with the closed triangular region $\mathrm{V}_{1} \mathrm{~V}_{2} \mathrm{~V}_{3}$.

Convex (or barycentric) coordinates admit of physical interpretation. The point $P$ is the center of mass for the discrete distribution of point masses $\alpha_{\mathfrak{t}}$ at $V_{1}$ for $i=1,2,3, \ldots, n, n+1$. The notions of balance and mechanical equilibrium permit us to discover the convex coordinates of various special points of triangles and tetrahedra and to relate the geometry of triangles to mechanics. [2]

Convex coordinates are quite versatile mathematical objects. Their invariance under affine transformations has many applications in geometry and their interpretation as lengths, areas, and volumes leads to a way to count partitions of the integers. [3]

Since $0 \leq \alpha_{2} \leq 1$ for each point of $S_{n}$, it has also seemed natural to seek probabilistic interpretations and applications for convex coordinates. Such interpretations and applications in one and two dimensions are the concerns of this paper. 
A RANDOM WALK ON A LINE SEGMENT. Let us consider a line $\ell$ on which there is defined a Cartesian coordinate system so that points $\mathrm{V}_{1}$ and $\mathrm{V}_{2}$ of $\ell$ have Cartesian coordinates $x=0$ and $x=1$, respectively. Next, let us consider $n-1$ points, $A_{1}, A_{2}, \ldots, A_{n-1}$, equally spaced between $V_{1}$ and $V_{2}$ as shown in Figure 1. For convenience in notation, let us give $V_{1}$ and $V_{2}$ the alternate names $A_{0}$ and $A_{n}$, respectively. The collection of $n$ segments $\overline{A_{i} A_{i+1}}, i=0,1,2, \ldots$, $\mathrm{n}-1$, and $n+1$ points $\mathrm{A}, \mathrm{j}=0,1,2, \ldots, \mathrm{n}$, constitutes a one dimensional graph. The convex coordinates of point $A_{i}$ with respect to $V_{1}=A_{0}$ and $V_{2}=A_{n}$ are $\left(\alpha_{1}, \alpha_{2}\right)=\left(1-\frac{i}{n}, \frac{i}{n}\right)$ for $\mathrm{i}=0,1,2, \ldots, \mathrm{n}$.

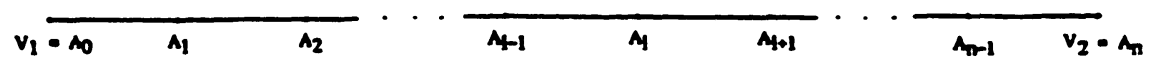

FIGURE 1. The One Dimensional Graph.

Now let us suppose that a random walk takes place on the one-dimensional graph. If the walk has reached $\mathrm{A}_{i}, \mathrm{i}=1,2, \ldots, \mathrm{n}-1$, the next step must be either to $\mathrm{A}_{1-1}$ with transition probability $p\left(A_{i} \rightarrow A_{i-1}\right)=\frac{1}{2}$ or to $A_{\mathrm{t}+1}$ also with transition probability $p\left(A_{i} \rightarrow A_{1+1}\right)=\frac{1}{2}$. End points $\mathrm{V}_{1}=A_{0}$ and $\mathrm{V}_{2}=A_{n}$ serve as traps. That is, $p\left(A_{0} \rightarrow A_{1}\right)=p\left(A_{n} \rightarrow A_{n-1}\right)=0$. The walk must continue until it reaches either $\mathrm{V}_{1}=A_{0}$ or $\mathrm{V}_{2}=A_{n}$.

Let $p\left(A_{i}\right)$ denote the probability that a walk starting from $A_{i}$ reaches endpoint $\mathrm{V}_{2}$. Then $p\left(A_{i}\right)$ must satisfy the equation

$$
\begin{aligned}
p\left(A_{i}\right) & =p\left(A_{i} \rightarrow A_{i-1}\right) p\left(A_{i}\right)+p\left(A_{i} \rightarrow A_{i+1}\right) p\left(A_{i+1}\right) \\
& =\frac{1}{2}\left(p\left(A_{i-1}\right)+\left(p\left(A_{i+1}\right)\right)\right.
\end{aligned}
$$

for $\mathrm{i}=1,2, \ldots, \mathrm{n}-1$, subject to the boundary conditions $p\left(A_{0}\right)=0, p\left(A_{n}\right)=1$. A function such as $p\left(A_{i}\right)$ is an example of a discrete harmonic function; and, if $p\left(A_{i}\right)$ satisfies both 1 and the boundary conditions, it does so uniquely. $[7,9]$

It is simple to show that $p\left(A_{i}\right)=\alpha_{2}$, the convex coordinate of $A_{i}$ with respect to $\mathrm{V}_{2}=A_{n}$, meets the requirements given above. Thus the probability that a walk starting at $A_{i}$ with terminate at $\mathrm{V}_{2}$ is $\alpha_{2}$. Likewise, the probability that the walk will terminate at $\mathrm{V}_{1}$ is $q\left(A_{i}\right)=1-p\left(A_{i}\right)=1-\alpha_{2}=\alpha_{1}$.

THE EXPECTED LENGTH OF THE ONE DIMENSIONAL RANDOM WALK. Each walk beginning at $A_{i}$ eventually terminates at $\mathrm{V}_{1}=A_{0}$ or $\mathrm{V}_{2}=A_{n}$. Let $E\left(A_{i}\right)$ denote the expected length of a walk as measured by the number of steps taken from starting point $A_{i}$ to either endpoint. Since the walk begins with a single step from interior point $A_{i}$ to either $A_{1-1}$ or $A_{i+1}$, we can write

$$
\begin{aligned}
E\left(A_{i}\right) & =p\left(A_{i} \rightarrow A_{i-1}\right) \cdot\left(1+E\left(A_{i-1}\right)\right) \\
& +p\left(A_{i} \rightarrow A_{i+1}\right) \cdot\left(1+E\left(A_{i+1}\right)\right) \\
& =\frac{1}{2}\left(E\left(A_{i-1}\right)+E\left(A_{i+1}\right)\right)+1 .
\end{aligned}
$$

Since a walk beginning at either endpoint will have zero length, our boundary conditions are that $E\left(A_{0}\right)=0$ and $E\left(A_{n}\right)=0$.

Suppose that both $E\left(A_{i}\right)$ and $E\left(A_{n}\right)$ satisfy 2 and the boundary conditions. Then $F\left(A_{1}\right)$ $=E\left(A_{i}\right)-E^{\prime}\left(A_{i}\right)$ satisfies 1 with boundary conditions $E\left(A_{0}\right)=E\left(A_{n}\right)=0$ and $F\left(A_{i}\right)=0$. Thus $F\left(A_{i}\right)$ is a discrete harmonic function and must be uniquely given by $F\left(A_{i}\right)=0$ for $\mathrm{i}=1,2, \ldots$, n. Therefore $E\left(A_{i}\right)=E^{\prime}\left(A_{i}\right)$, and any function satisfying 2 while vanishing at the endpoints is the unique expected length of the walk. 
Since $E\left(A_{i}\right)=n^{2} \alpha_{1} \alpha_{2}$ satisfies 2 and the convex coordinates of $\mathrm{V}_{1}, \mathrm{~V}_{2}$ are $(1,0),(0,1)$, respectively, we have the expected length. That is, the expected length of a random walk starting from $A_{1}$ on the one dimensional or linear graph of $n+1$ points and $n$ segments is $E\left(A_{\mathfrak{q}}\right)$ $=n^{2} \alpha_{1} \alpha_{2}$ where $\alpha_{1}, \alpha_{2}$ are the convex coordinates of $A_{2}$.

A RANDOM WALK ON A TRIANGLE. Consider the closed triangular region named by its vertices as $V_{1} V_{2} V_{3}$. For convenience, we take the triangle to be equilateral. We consider each side to be a copy of the one dimensional graph of $n+1$ equally spaced points and $n$ connecting segments described above. If a line is drawn within $\mathrm{V}_{1} \mathrm{~V}_{2} \mathrm{~V}_{3}$ parallel to side $\overline{\mathrm{V}_{i} \mathrm{~V}_{3}}$, then each point of the line has the same convex coordinate $\alpha_{k}, k \neq i, j$. Suppose, then, that three lines are drawn so that each is parallel to a different side of the triangle and so that the three lines are concurrent. Each is a line of constant convex coordinate with respect to a different vertex. Thus the convex coordinates for the point $P$ common to the three lines can be read from the points at which the lines intersect the side of the triangle as indicated in Figure 2.

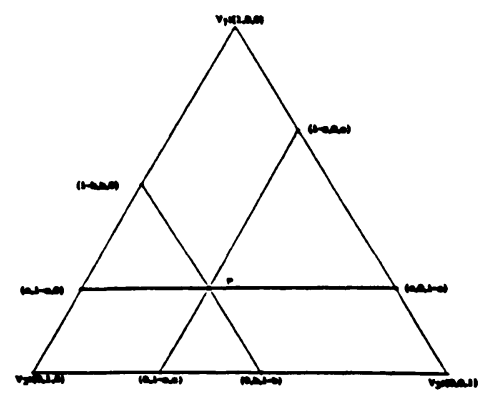

FIGURE 2. The Convex Coordinates of $P:(\mathrm{a}, \mathrm{b}, \mathrm{c})$.

Now let us draw three sets of $n-1$ lines each parallel to one of the three sides so that the lines divide the sides into $n$ congruent segments. In Figure 3, we show the three sets of lines for the case in which $n=6$. The interior intersection point $P$ has six adjacent points.

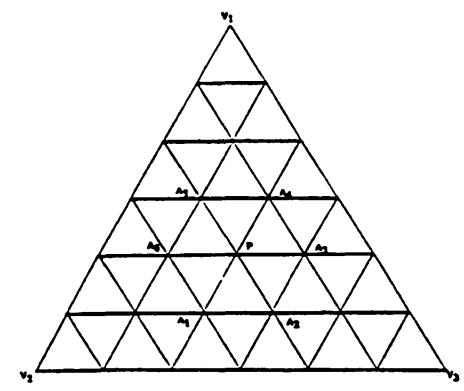

FIGURE 3. The Triangular Graph for $n=6$.

The array of points and segments constitutes a two dimensional, triangular graph upon which we will consider a random walk. Each step is a move between adjacent points of the graph along the segment connecting them. If we denote the points adjacent to interior point $P$ by $A_{1}$ for $\mathrm{i}=1,2,3,4,5,6$, we can state the following rules for the random walk.

1. The probability of a move from $P$ to $A_{i}$ is given by $p\left(P \rightarrow A_{i}\right)=\frac{1}{6}$.

2. Once the walk reaches a boundary point, the walk must remain on the boundary.

3. The vertices of the triangle serve as traps. The walk must terminate once it reaches a vertex. 
4. The probability of a move from any boundary point other than a vertex to either of its two neighboring boundary points is $\frac{1}{2}$. That is, once the walk has reached a side of the triangle, the walk becomes a one dimensional walk as described above.

Let us now suppose that Figure 3 represents the general case for the triangular graph with $n \geq 3$. Then, if the convex coordinates of interior point $P$ are $\alpha(P)=\left(\alpha_{1}, \alpha_{2}, \alpha_{3}\right)$, the convex coordinates of its nearest neighboring points are

$$
\begin{array}{ll}
\alpha\left(A_{1}\right)=\left(\alpha_{1}-\frac{1}{n}, \alpha_{2}+\frac{1}{n}, \alpha_{3}\right), & \alpha\left(A_{2}\right)=\left(\alpha_{1}-\frac{1}{n}, \alpha_{2}, \alpha_{3}+\frac{1}{n}\right), \\
\alpha\left(A_{3}\right)=\left(\alpha_{1}, \alpha_{2}-\frac{1}{n}, \alpha_{3}+\frac{1}{n}\right), & \alpha\left(A_{4}\right)=\left(\alpha_{1}+\frac{1}{n}, \alpha_{2}-\frac{1}{n}, \alpha_{3}\right), \\
\alpha\left(A_{5}\right)=\left(\alpha_{1}+\frac{1}{n}, \alpha_{2}, \alpha_{3}-\frac{1}{n}\right), & \alpha\left(A_{6}\right)=\left(\alpha_{1}, \alpha_{2}+\frac{1}{n}, \alpha_{3}-\frac{1}{n}\right) .
\end{array}
$$

By direct computation, we see that

If $P$ is a boundary point as shown in Figure 4 , we also have

$$
\alpha(P)=\left(\alpha_{1}, \alpha_{2}, \alpha_{3}\right)=\frac{1}{6} \sum_{i=1}^{6} \alpha\left(A_{1}\right)
$$

$$
\alpha(P)=\frac{1}{2}\left(\alpha\left(A_{1}\right)+\alpha\left(A_{2}\right)\right) \text {. }
$$

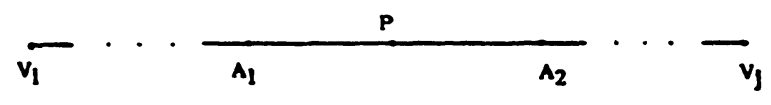

FIGURE 4. Point $P$ on Side $\overline{V_{i} V_{j}}$.

Let $p(P)$ denote the probability that a random walk starting from point $P$ of the triangular graph will reach vertex $\mathrm{V}_{1}$. The function $p(P)$ is again a discrete harmonic function. It satisfies the condition

$$
p(P)=\sum_{i=1}^{6} p\left(P \rightarrow A_{i}\right) \cdot p\left(A_{i}\right)=\frac{1}{6} \sum_{i=1}^{6} p\left(A_{i}\right)
$$

for each interior point $P$. On the boundary $p(P)=\frac{1}{2}\left(p\left(A_{1}\right)+p\left(A_{2}\right)\right)$ with the notation taken from Figure 4.

Equation 3 and the results for the one dimensional walk imply that $p(P)=\alpha_{1}$. That is, the probability of reaching $V_{1}$ starting from point $P$ is $\alpha_{1}$. Likewise, the probabilities of reaching $V_{2}$ and $V_{3}$ are $\alpha_{2}$ and $\alpha_{3}$, respectively.

The walk can be extended to graphs on tetrahedron $V_{1} V_{2} V_{3} V_{4}$. Each face contains a triangular graph and each edge contains a linear graph. The probability of a random walk's reaching vertex $\mathrm{V}_{i}, \mathrm{i}=1,2,3,4$ from starting point $P$ is $\alpha_{i}$, the convex coordinate of $P$ with respect to vertex $V_{\imath}$. The walk can be extended inductively to graphs on the $k$-simplex, each face of which is a $(k-1)$-simplex.

ELECTRICAL APPLICATION 1. For each random walk there exists an electrical potential problem having the same solution with the only difference between the problems being the physical meaning of the variables. [4]

Imagine that the triangular grid of Figure 5 is an electrical network of identical resistors in which each segment connecting adjacent points has resistance $R$. Let the potential at $\mathrm{V}_{1}$ be maintained at 1 volt while all points of $\overline{V_{2} V_{3}}$ are held at zero potential. The potentials on sides $\overline{V_{1} V_{2}}$ and $\overline{V_{1} V_{3}}$ decrease linearly from 1 to 0 volts. Thus the boundary conditions on potential match those of the random walk.

At points $P$ and $A_{t}$ the potentials may be represented by $v(P)$ and $v\left(A_{\mathrm{t}}\right)$, respectively. Since the algebraic sum of currents into and out of $P$ must be zero, we can write 


$$
\sum_{i=1}^{6}\left(\frac{v\left(A_{i}\right)-v(P)}{R}\right)=0
$$

or $v(P)=\frac{1}{6} \sum_{i}^{6} v\left(A_{\mathrm{i}}\right)$. Thus the average value property given by Equation 3 is satisfied. We can conclude that the electrical potential at point $P$ is $\alpha_{1}$.

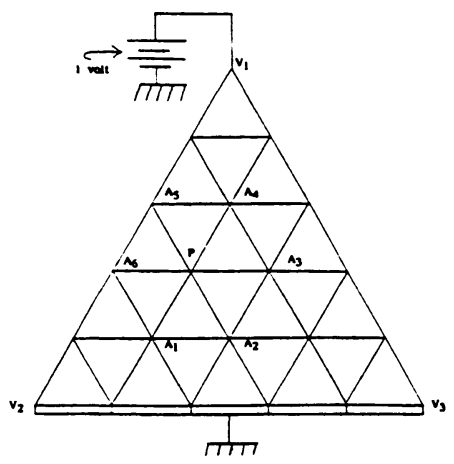

Figure 5. An Electrical Network.

THE EXPECTED LENGTH OF THE TRIANGULAR RANDOM WALK. Let us suppose that a random walk starts from point $P$ of the triangular graph and terminates at one of the vertices. Let us denote the expected length of the walk by $E(P)$.

If $P$ is an interior point having nearest neighbors $A_{z}, \mathrm{i}=1,2,3,4,5,6$, it follows that

$$
E(P)=\sum_{i=1}^{6} \frac{1}{6}\left(1+E\left(A_{i}\right)\right)=1+\frac{1}{6} \sum_{i=1}^{6} E\left(A_{i}\right)
$$

If $P$ is a boundary point with boundary neighbors $A_{1}, A_{2}$ on $\overline{V_{1} V_{j}}$, we already know that $E(P)=n^{2} \alpha_{i} \alpha$, satisfies $E(P)=1+\frac{1}{2}\left(E\left(A_{1}\right)+\left(E\left(A_{2}\right)\right)\right.$. Furthermore, $E\left(\mathrm{~V}_{i}\right)=E\left(\mathrm{~V}_{j}\right)=0$.

Since $E(P)=n^{2}\left(\alpha_{1} \alpha_{2}+\alpha_{1} \alpha_{3}+\alpha_{2} \alpha_{3}\right)$ satisfies Equation 4 and also reduces to the boundary solution, it follows that we have discovered the expected value of the length of the random walk.

That is, the expected length of a walk starting from point $P$ of the triangular graph is $E(P)=n^{2}\left(\alpha_{1} \alpha_{2}+\alpha_{1} \alpha_{3}+\alpha_{2} \alpha_{3}\right)$ where $\alpha_{1}, \alpha_{2}, \alpha_{3}$ are convex coordinates of $\dot{P}$ with respect to $\mathrm{V}_{1}, \mathrm{~V}_{2}, \mathrm{~V}_{3}$ in that order.

AN EXAMPLE. Let $n=12$. The centroid of the triangle has convex coordinates $\left(\frac{1}{3}, \frac{1}{3}, \frac{1}{3}\right)$ and is a point of the graph.

The probability that a walk starting from the centroid will reach a particular vertex is $\frac{1}{3}$ as is intuitively obvious. The expected length of the walk is $\left(12^{2}\right)\left(\frac{1}{3} \cdot \frac{1}{3}+\frac{1}{3} \cdot \frac{1}{3}+\frac{1}{3} \cdot \frac{1}{3}\right)=48$ steps.

COMMENTS ON BOUNDARY CONDITIONS. A natural problem concerns the construction of discrete harmonic functions on the interior points of the triangular graph when the functions are required to meet arbitrarily set boundary conditions. We consider the problem with a special case.

Let us change the fourth of our rules for random walks on triangles by supposing that the entire boundary of $\Delta V_{1} V_{2} V_{3}$ serves as a trap. In other words, the random walk must come to a halt whenever it reaches a boundary point. Let us find or indicate how to find discrete harmonic functions in terms of $\alpha_{1}, \alpha_{2}$, and $\alpha_{3}$ in closed form which will give the probability of reaching a point of side $\overline{V_{2} V_{3}}$ from any point $P:\left(\alpha_{1}, \alpha_{2}, \alpha_{3}\right)$ of the triangular graph. Again, we 
denote the desired probability by $p(P)$.

Since the vertices $V_{1}, V_{2}, V_{3}$ cannot be reached directly from any interior point of the array, we can exclude them from our calculations. In Figure 6, we indicate the triangular graph with $\mathrm{V}_{1}, \mathrm{~V}_{2}, \mathrm{~V}_{3}$ removed.

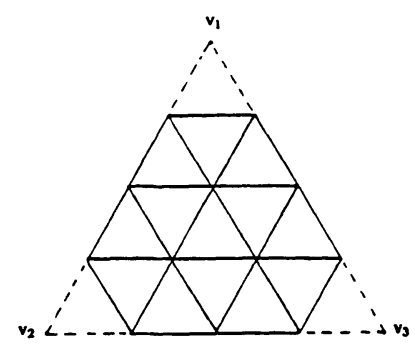

Figure 6. $\Delta \mathrm{V}_{1} \mathrm{~V}_{2} \mathrm{~V}_{3}-\left\{\mathrm{V}_{1}, \mathrm{~V}_{2}, \mathrm{~V}_{3}\right\}$.

The boundary conditions for $p(P)$ are $p(P)=0$ if $P \epsilon \overline{V_{1} V_{2}} \cup \overline{V_{1} V_{3}}-\left\{V_{1}, V_{2}, V_{3}\right\}$ and $p(P)=1$ if $P \epsilon \overline{V_{2} V_{3}}-\left\{\mathrm{V}_{2}, V_{3}\right\}$. Once we find a function $p(P)$ satisfying both these boundary conditions and the average value property given by 3 , that probability function will be unique.

However, the forms of the functions will not be unique. For example, $p(P)=\alpha_{1}+\alpha_{2}$ is harmonic, yet it has the different, but equivalent, form $p(P)=1-\alpha_{3}$. Furthermore, since the functions need be evaluated only at a finite number of points, various combinations of continuous functions may take on the same values.

A bit of calculation indicates that $p(P)=\cos n \pi \alpha_{1} \cos n \pi \alpha_{2} \cos n \pi \alpha_{3}$ satisfies the average value property, but the value of $p(P)$ is 1 at all interior points of the graph if $n$ is even and -1 if $n$ is odd.

This example involving the trigonometric functions suggests that we investigate products of exponential functions in our search for other, more useful, harmonic functions. Therefore, suppose that $f(P)=a^{n \alpha_{1}} b^{n \alpha_{2}} c^{n \alpha_{3}}$ for nonnegative real numbers $a, b, c$. We can rewrite the function as $f(P)=a^{n \alpha_{1}} b^{n \alpha_{2}} c^{n-n \alpha_{1}-n \alpha_{2}}=c^{n}\left(\frac{a}{b}\right)^{n \alpha_{1}}\left(\frac{a}{b}\right)^{n \alpha_{2}}$ implying that we need only consider products of the form $p(P)=a^{n \alpha_{1}} b^{n \alpha_{2}}$.

If $p(P)=a^{n \alpha_{1}} b^{n \alpha_{2}}$ is to satisfy the average value property, we require that

$$
\begin{aligned}
& a^{n\left(\alpha_{1}-\frac{1}{n}\right)} b^{n\left(\alpha_{2}-\frac{1}{n}\right)}+a^{n\left(\alpha_{1}-\frac{1}{n}\right)} b^{n \alpha_{2}}+a^{n \alpha_{1}} b^{n\left(\alpha_{2}-\frac{1}{n}\right)} \\
& \quad+a^{n\left(\alpha_{1}+\frac{1}{n}\right)} b^{n\left(\alpha_{2}-\frac{1}{n}\right)}+a^{n\left(\alpha_{1}+\frac{1}{n}\right)} b^{n \alpha_{2}}+a^{n \alpha_{1}} b^{n\left(\alpha_{2}+\frac{1}{n}\right)}=6 a^{n \alpha_{1}} b^{n \alpha_{2}} .
\end{aligned}
$$

Simplifying this equation, we find that $a^{-1} b+a^{-1}+b^{-1}+a b^{-1}+a+b=6$ or $(a+1) b^{2}+\left(a^{2}-6 a+1\right) b+\left(a^{2}+a\right)=0$.

In order that $b$ take on real values, it is necessary that the discriminant $D$ be nonnegative. The discriminant takes the simplified form $D=(a-1)^{2}\left(a^{2}-14 a+1\right)$ implying that $a$ should be chosen from $(-\infty, 7-4 \sqrt{3}] \cup[7+4 \sqrt{3}, \infty)$. In addition, neither $a$ nor $b$ may be zero.

Since we intend to use sums of functions of the form (constant) $\cdot a^{n \alpha_{1}} b^{n \alpha_{2}}$ to satisfy the boundary conditions of our problem, let us choose $a$ and $b$ to yield as simple calculations as possible. To that end, we list all of the admissible values of $a$ that give discriminants which are perfect squares. 


\begin{tabular}{|c|l|r|}
\hline$a$ & $D$ & $b$ \\
\hline-6 & 5929 & $\frac{2}{5}$ or 15 \\
14 & 169 & $-\frac{21}{5}$ or $-\frac{10}{5}$ \\
15 & 3136 & -6 or $-\frac{5}{2}$ \\
20 & 43681 & $-\frac{5}{3}$ or $-\frac{12}{7}$ \\
\hline
\end{tabular}

Table 1. Values of $a, D, b$.

Since the roles of $a$ and $b$ may be interchanged, we can construct fifteen different functions in $\alpha_{1}$ and $\alpha_{2}$ which have one integral base while the other base is rational. In addition, we can use the pairs of convex coordinates $\alpha_{1}, \alpha_{3}$ or $\alpha_{2}, \alpha_{3}$ as independent variables. Of course, $a$ and $b$ need not be rational, but our immediate purpose is to produce simple examples.

In Figure 7 , we show the simple graph for $n=3$. The probability that a walk starting at $P$, the only interior point, will reach a point of $\overline{V_{2} V_{3}}$ is clearly $\frac{1}{3}$. However, we use this graph as a test case to display the process for constructing a discrete harmonic function giving the desired probability.

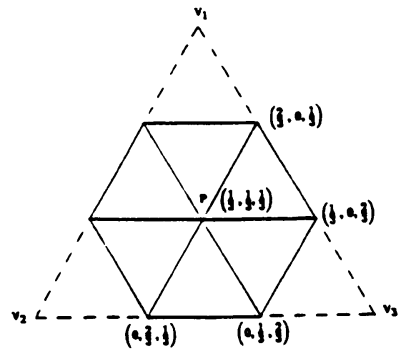

Figure 7. The Case for $n=3$.

The problem is symmetric with respect to $\alpha_{2}$ and $\alpha_{3}$. Since sums of harmonic functions are harmonic, let us find constants $A, B, C$, and $D$ so that

$$
p(P)=A\left[15^{3 \alpha_{1}}(-6)^{3 \alpha_{2}}+15^{3 \alpha_{1}}(-6)^{3 \alpha_{3}}\right]+B \alpha_{1}+C \alpha_{2}+D \alpha_{3}
$$

vanishes if $\left(\alpha_{1}, \alpha_{2}, \alpha_{3}\right) \in\left\{\left(\frac{2}{3}, 0, \frac{1}{3}\right),\left(\frac{1}{3}, 0, \frac{2}{3}\right)\right\}$ and has the value 1 if $\left(\alpha_{1}, \alpha_{2}, \alpha_{3}\right) \epsilon\left\{\left(0, \frac{2}{3}, \frac{1}{3}\right)\right.$, $\left.\left(0, \frac{1}{3}, \frac{2}{3}\right)\right\}$. Note that symmetry implies that if $p(P)$ vanishes on $\overline{V_{1} V_{3}}$, it will also vanish on

$\overline{V_{1} V_{2}}$. Substitution of appropriate values of $\alpha_{1}, \alpha_{2}, \alpha_{3}$ into Equation 5 yields the following linear equations:

$$
\begin{aligned}
-3375 A+2 B+D & =0 \\
1665 A+B+2 D & =0 \\
90 A+C+2 D & =3, \\
90 A+2 C+D & =3 .
\end{aligned}
$$

Solving these equations, we obtain $A=-1 / 2205, B=-187 / 147, C=D=149 / 147$.

Checking our work by evaluation of the probability function with these coefficients at $\left(\alpha_{1}, \alpha_{2}, \alpha_{3}\right)=\left(\frac{1}{3}, \frac{1}{3}, \frac{1}{3}\right)$, we obtain $\frac{1}{3}$ as desired.

In Figure 8, we show the case for $n=4$. 


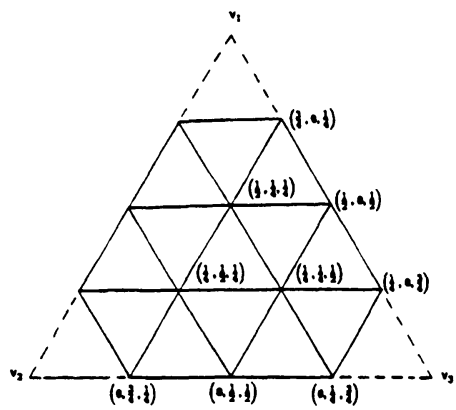

Figure 8. The Case for $n=4$.

Let $y$ represent the probability of reaching $\overline{V_{2} V_{3}}$ form $\left(\frac{1}{2}, \frac{1}{4}, \frac{1}{4}\right)$. By symmetry, the probabilities of reaching $\overline{V_{2} V_{3}}$ from $\left(\frac{1}{4}, \frac{1}{2}, \frac{1}{4}\right)$ and $\left(\frac{1}{4}, \frac{1}{4}, \frac{1}{2}\right)$ are the same; and we denote that probability by $x$. The average value property implies that

Thus $x=\frac{3}{7}, y=\frac{1}{7}$.

$$
\frac{0+0+1+1+x+y}{6}=x \quad \text { and } \quad \frac{0+0+0+0+x+x}{6}=y .
$$

Now let us construct $p(P)$ and then compare our function values with $x$ and $y$. Recalling that the form of $p(P)$ is not unique, we simply choose our form for reasons of convenience. Let

$$
\begin{aligned}
p(P) & =A\left[15^{4 \alpha_{1}}(-6)^{4 \alpha_{1}}+15^{4 \alpha_{1}}(-6)^{4 \alpha_{3}}\right] \\
& +B\left[14^{4 \alpha_{1}}\left(-\frac{21}{5}\right)^{4 \alpha_{2}}+14^{4 \alpha_{1}}\left(-\frac{21}{5}\right)^{4 \alpha_{3}}\right] \\
& +C\left[15^{4 \alpha_{1}}\left(-\frac{5}{2}\right)^{4 \alpha_{2}}+15^{4 \alpha_{1}}\left(-\frac{5}{2}\right)^{4 \alpha_{3}}\right] \\
& +D \alpha_{1}+E \alpha_{2}+F \alpha_{3} .
\end{aligned}
$$

Using the convex coordinates of points from $\overline{V_{1} V_{3}} \cup \overline{V_{2} V_{3}}-\left\{V_{1}, V_{2}, V_{3}\right\}$ and noting that $E=F$, we can write the equations

$$
\begin{aligned}
-16874 A-8780.8 B-5062.5 C+.75 D+.25 F & =0 \\
8325 A+3653.44 B+1631.25 C+.5 D+.5 F & =0 \\
-3225 A-1023.232 B-219.375 C+.25 D+.75 F & =0 \\
-22 A-78.288 B-18.125 C+F & =1 \\
72 A+35.28 B+12.5 C+F & =1
\end{aligned}
$$

A computer program to implement a Gaussian elimination procedure yields $A=1.425 \times$ $10^{-4}, B=1.029 \times 10^{-4}, C=-8.189 \times 10^{-4}, D=-1.449$, and $E=F=0.996$. Evaluating $p(P)$ at $\left(\frac{1}{2}, \frac{1}{4}, \frac{1}{4}\right)$ and $\left(\frac{1}{4}, \frac{1}{2}, \frac{1}{4}\right)$, we obtain .141 and .422 whose differences from $\frac{1}{7}$ and $\frac{3}{7}$ are easily explained by round-off errors in computation.

We conclude that linear combinations of functions of the form $a^{n \alpha_{1}} b^{n \alpha_{2}}$, $a^{n \alpha_{1}} b^{n \alpha_{3}}, a^{n \alpha_{2}} b^{n \alpha_{3}}$, and $\alpha_{1}, \alpha_{2}, \alpha_{3}$ will produce discrete harmonic functions satisfying arbitrarily set boundary conditions for triangular random walks. Unfortunately, the computations do not seem anesthetically pleasing, but they do generalize the subject.

ELECTRICAL APPLICATION 2. Let us consider the network below which is derived from the graph shown in Figure 8. All segments have the same resistance $R$. All points of sides 
$\overline{V_{1} V_{2}}-\left\{V_{1}, V_{2}\right\}$ and $\overline{V_{1} V_{2}}-\left\{V_{1}, V_{2}\right\}$ are maintained at a potential of +1 volt.

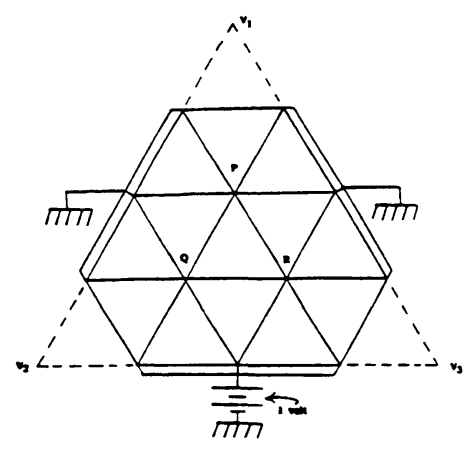

Figure 9. Another Electrical Network. and $\frac{3}{7}$, respectively.

It follows from our last computations that the potentials at points $P, Q$, and $R$ are $\frac{1}{7}, \frac{3}{7}$,

THE EXPECTED LENGTH OF THE RANDOM WALK WHEN THE BOUNDARIES ARE TRAPS. In the case that all points of the boundary of triangular graph $\mathrm{V}_{1} \mathrm{~V}_{2} \mathrm{~V}_{3}$ serve as traps, the expected length of a random walk from interior point $P:\left(\alpha_{1}, \alpha_{2}, \alpha_{3}\right)$ is given by $E(P)$ $=3 n^{2} \alpha_{1} \alpha_{2} \alpha_{3}$. This result follows from the fact that $E(P)=\frac{1}{6} \sum_{i=1}^{6} E\left(A_{i}\right)+1=3 n^{2} \alpha_{1} \alpha_{2} \alpha_{3}$. Again, the average is taken over adjacent points $A_{\mathfrak{l}}, i=1,2,3,4,5,6$. The claim can be verified quite easily by direct computation over the six points adjacent to $P$ in the graph having $n+1$ points on each side. It is also clear that $E(P)$ vanishes on the boundary of the triangle since at least one convex coordinate must be zero at any boundary point. The result is unique by arguments similar to those given in the one dimensional case.

In the simple case with $n=3, E(P)=1$ for the walk starting from the centroid. If $n=$ 6 and $P$ is again the centroid with convex coordinates $\left(\frac{1}{3}, \frac{1}{3}, \frac{1}{3}\right)$, then $E(P)=E(P)=4$. If $n=12$ and $P$ is the centroid, then $E(P)=16$.

FURTHER COMMENTS ON EXPECTED LENGTHS. Again, it is the result of straightforward computation that, for interior point $P:\left(\alpha_{1}, \alpha_{2}, \alpha_{3}\right)$, each of the following functions $n^{2} \alpha_{1} \alpha_{2}, n^{2} \alpha_{2} \alpha_{3}, n^{2} \alpha_{1} \alpha_{3}$, and $n^{2} \alpha_{1} \alpha_{2} \alpha_{3}$ satisfies the average value property $\frac{1}{6} \sum_{i=1}^{6}$ $f\left(A_{i}\right)+\frac{1}{3}=f(P)$.

It follows that $E(P)=n^{2}\left(a \alpha_{1} \alpha_{2}+\mathrm{b} \alpha_{2} \alpha_{2}+\mathrm{c} \alpha_{1} \alpha_{3}+\mathrm{d} \alpha_{1} \alpha_{2} \alpha_{3}\right)$ satisfies Equation4 and becomes a candidate for an expected length if the real coefficients, a,b,c,d, sum to 3 .

For example, if $a=b=c=1, d=0$, we have the expected length for the walk in which the vertices are the only traps and for which original boundary condition 4 holds.

If $a=b=c=0, d=3$, we have the expected length if all boundary points serve as traps.

Suppose that all points of $\overline{V_{1} V_{2}} \cup \overline{V_{1} V_{3}}$ serve as traps but that boundary condition 4 holds true on $\overline{\mathrm{V}_{2} \mathrm{~V}_{3}}$. Then $E(P)$ is given by $\mathrm{a}=0, \mathrm{~b}=1, \mathrm{c}=0, \mathrm{~d}=2$ since such a function uniquely satisfies the boundary conditions.

In similar fashion, other values of $a, b, c$, and $d$ may be sought to satisfy various conditions for terminating the walk on the boundary. 


\section{REFERENCES}

1. GRAY, J. "Möbius' Geometrical Mechanics," in Möbius and His Band (J. Fauvel, R. Flood, and R. Wilson, Editors), Oxford University Press, New York, pp. 78-103, 1993.

2. HANSNER, M. "The Center of Mass and Affine Geometry," The American Mathematical Monthly $\underline{69}$ (October 1962), 724-737.

3. BOYD, J.N., and RAYCHOWDHURY, P.N. "Counting Partitions of the Integers with Convex Coordinates," Bulletin of Number Theory XV (1991), 28-37.

4. DOYLE, P.G. and SNELL, J.L., "Random Walks and Electrical Networks" Mathematical Association of America, Washington, D.C. (1984). 


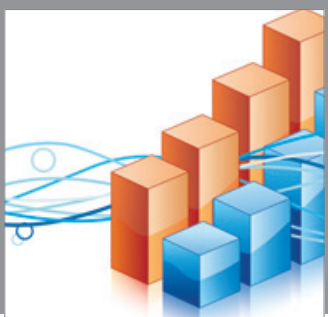

Advances in

Operations Research

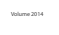

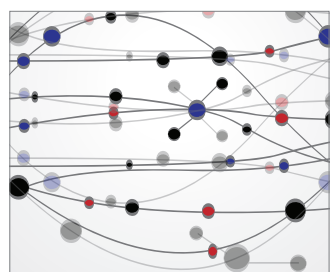

\section{The Scientific} World Journal
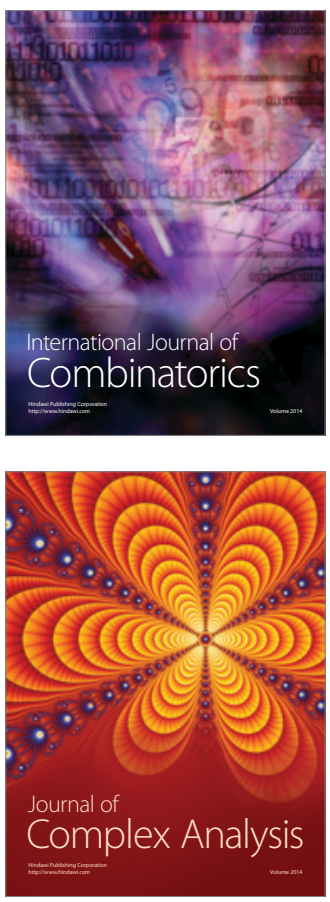

International Journal of

Mathematics and

Mathematical

Sciences
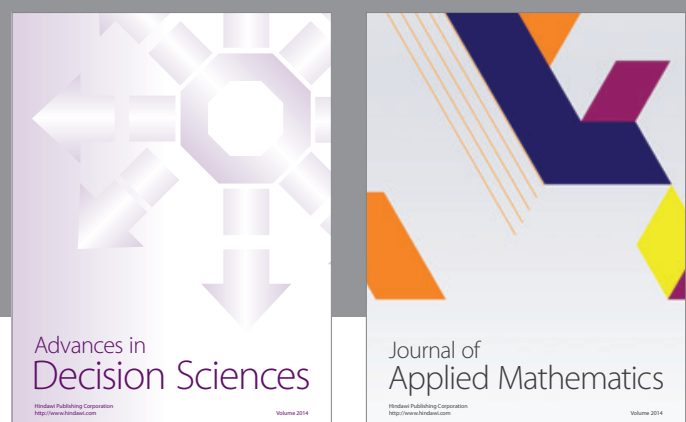

Journal of

Applied Mathematics
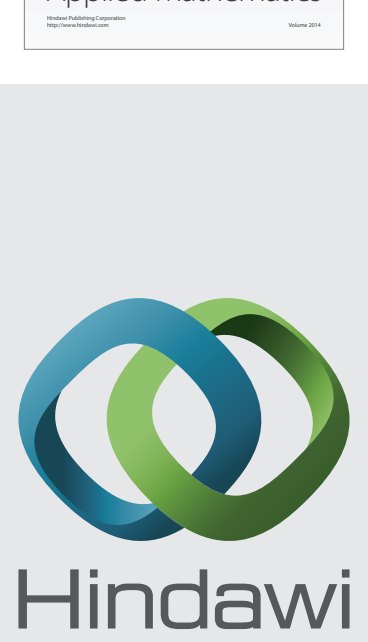

Submit your manuscripts at http://www.hindawi.com
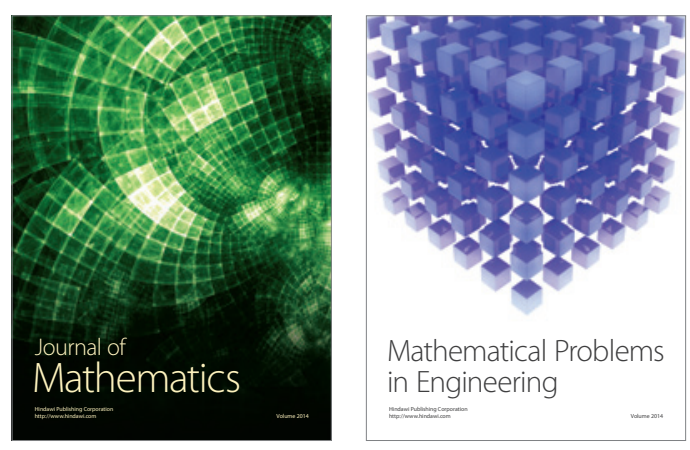

Mathematical Problems in Engineering
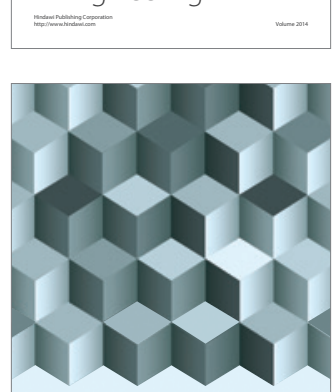

Journal of

Function Spaces
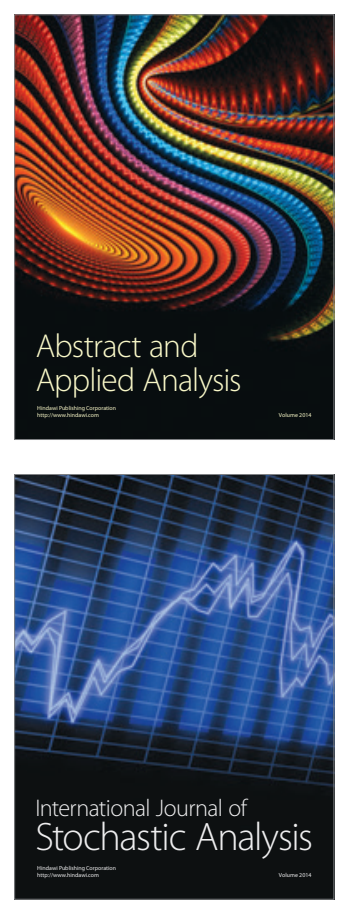

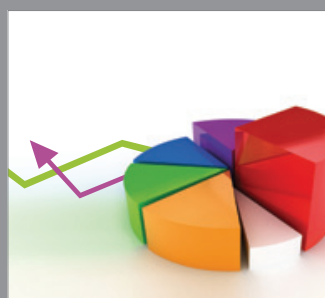

ournal of

Probability and Statistics

Promensencen
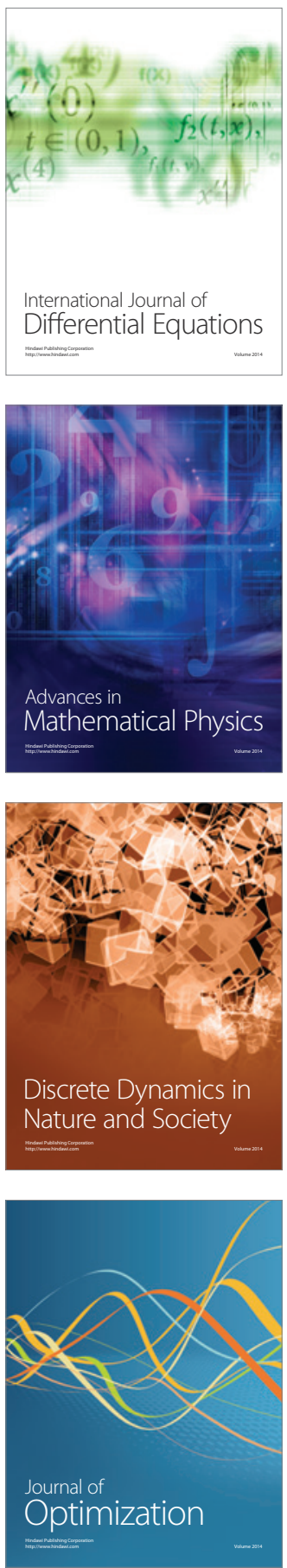